\title{
QUEBRANDO TABUS: OS FATORES QUE LEVAM O SUICÍDIO ENTRE UNIVERSITÁRIOS.
}

\author{
Hélida Maravilha Dantas e Sousa Almeida, Universidade Federal de Campina Grande \\ (UFCG), \\ helidacaico@hotmail.com \\ Maria Heloisa Alves Benedito, Universidade Federal de Campina Grande (UFCG), \\ heloisaalvesb@gmail.com \\ Sávio Benvindo Ferreira, Universidade Federal de Campina Grande (UFCG), \\ saviobenvindo@gmail.com
}

\begin{abstract}
RESUMO
Os universitários são uma classe de jovens que possui uma realidade muito específica, por causa do ambiente acadêmico e fatores sociais, e vem chamando a atenção de muitos pesquisadores por apresentarem um aumento significativo no desenvolvimento das doenças neuropsíquicas, em destaque a depressão, que em casos graves levam ao suicídio. Essas enfermidades estão cada vez mais provocando um impacto na saúde mundial. Esse trabalho tem como objetivo identificar os fatores associados ao suicídio em universitários, por meio de uma pesquisa bibliográfica realizada em julho de 2017, com artigos científicos em inglês, português e espanhol, sendo selecionados 11 para compor o estudo. Os resultados encontrados foram que fatores intrínsecos a instituição de ensino superior como também fatores pessoais podem causar a depressão evoluindo para o suicídio desses estudantes. Portanto, é de extrema importância a identificação desses fatores pois assim será possível a realização de medidas de auxílio e prevenção pelas instituições de ensino superior, para abrandar e posteriormente sanar essa enfermidade social que vem destruindo a vida de muitos jovens.
\end{abstract}

PALAVRAS-CHAVE: Suicídio; universitários; fatores.

\section{BREAKING TABOOS: THE FACTORS THAT LEAD TO SUICIDE AMONG COLLEGE STUDENTS.}

\begin{abstract}
University students are a class of young people who have a very specific reality, because of the academic environment and social factors, and has attracted the attention of many researchers for presenting a significant increase in the development of neuropsychic diseases, in particular depression, which in cases Lead to suicide. These diseases are increasingly having an impact on global health. This work aims to identify the factors associated with suicide in university students, through a bibliographic research conducted in July 2017, with scientific articles in English, Portuguese and Spanish, being selected 11 to compose the study. The results found were that factors intrinsic to the institution of higher education as well as personal factors can cause depression evolving to the suicide of these students. Therefore, it is
\end{abstract}


extremely important to identify these factors, since it will be possible to carry out preventive and preventive measures by higher education institutions to slow down and later remedy this social disease that has been destroying the lives of many young people.

KEYWORDS: Suicide; university students; factors

\section{ROMPIENDO TABÚES: LOS FACTORES QUE LLEVAN EL SUICIDIO ENTRE UNIVERSITARIOS.}

\section{RESUMEN}

Los universitarios son una clase de jóvenes que posee una realidad muy específica, debido al ambiente académico y factores sociales, y viene llamando la atención de muchos investigadores por presentar un aumento significativo en el desarrollo de las enfermedades neuropsíquicas, en destaque la depresión, que en Los casos graves conduce al suicidio. Estas enfermedades están cada vez más provocando un impacto en la salud mundial. Este trabajo tiene como objetivo identificar los factores asociados al suicidio en universitarios, a través de una bibliografía bibliográfica realizada en julio de 2017, con artículos científicos en inglés, portugués y español, siendo seleccionados 11 para componer el estudio. Los resultados encontrados encontraron que factores intrínsecos a la investigación de enseñanza superior como también factores personales pueden causar la depresión evolucionando hacia el suicidio de los estudiantes. Por lo tanto, es de extrema importancia la identificación de los factores, así será posible la realización de medidas de ayuda y prevención por las instituciones de enseñanza superior, para ralentizar y posteriormente sanar esa enfermedad social que viene destruyendo la vida de muchos jóvenes.

PALABRAS CLAVES: Suicidio; Universitarios; Los factores.

\section{INTRODUÇÃO}

A estabilidade emocional de um indivíduo é essencial pois reflete em vários aspectos da vida, tanto social quanto na saúde individual, portanto, quando há uma alteração nesse equilíbrio alterará a qualidade de vida do indivíduo. Os jovens constituem um grupo social com características específicas, e por estarem saindo da adolescência e entrando na idade adulta, apresentam muitas vezes uma cobrança interna grande principalmente na forma como percebem a vida e uma externa representada em como a sociedade o considera. A cobrança sociocultural que essa classe sofre é evidente e ainda é um tabu na atualidade, o que acaba, diversas vezes, repercutindo em suas atitudes, os levando a um estilo de vida prejudicial à saúde em vários aspectos. 
Os universitários nessa faixa etária são uma classe específica que frequentemente lidam com desafios particulares, por estarem inseridos em um ambiente acadêmico em que sofrem grande pressão psicossocial, onde estão expostos a exigências constantes, que por vezes acabam levando a disfunções no seu bem-estar, podendo originar vários processos patológicos, inclusive os neuropsíquicos. Este problema é relatado por Cremasco e Baptista (2017) em sua pesquisa, que revela um percentual de $15 \%$ a $25 \%$ dos estudantes de graduação desenvolvem algum tipo de transtorno mental durante a sua formação, sendo a depressão um dos transtornos mais prevalentes.

Portanto esse estudo tem como finalidade identificar os fatores associados ao suicídio em universitários, possibilitando a não só profissionais da saúde, mas a sociedade como um todo manter-se informada a respeito dessa problemática, podendo posteriormente promover a elaboração de atividades que previnam esse tipo de fatalidade entre os estudantes universitários.

\section{METODOLOGIA}

Caracteriza-se por uma revisão integrativa da literatura, estruturada com abordagem qualitativa para a identificação de artigos científicos que abordassem a temática exposta. $\mathrm{O}$ levantamento bibliográfico foi realizado no mês de julho de 2017 nas bases de dados Latin American Literature in Health Sciences (LILACS), Indice Bibliográfico Español de Ciencias de la Salud (IBECS), Literatura Internacional em Ciências da Saúde (MEDLINE), Index Psi Periódicos Técnico-Científicos e Scientific Eletronic Library Online (SCIELO), via Biblioteca Virtual de Saúde, utilizando como título, resumo e assunto "universitários" "suicídio" e o operador booleano AND. Foram encontrados 233 resultados. Para a classificação, o período de publicação varia de 2014 a 2017, artigos disponíveis na íntegra, nos idiomas português, espanhol e inglês que abordavam a temática proposta. Os critérios de exclusão foram artigos duplicados, incompatíveis com a temática descrita, teses, relatos de caso, dissertações e revisões bibliográficas. A pesquisa foi constituída por 33 publicações incluídas que abordavam o tema exposto, dos quais foram selecionados para compor o estudo 11 artigos, sendo 2 em inglês, 6 em espanhol e 3 artigos em português, considerando os critérios de exclusão. 


\section{REVISÃO DE LITERATURA}

\section{Depressão e suicídio: sintomas, sinais e fatores}

A depressão e o suicídio são problemas globais, que atinge indivíduos de várias faixas etárias, tornando isso uma grande questão social e um agravante a saúde pública, Dados da Organização Mundial da Saúde (2012) reforçam que a depressão é um agente frequentemente encontrado na sociedade em geral, e estima que em 2030 será a principal razão pela incapacidade do mundo.

O Manual Diagnóstico e Estatístico de Transtornos Mentais ([DSM-5]; APA, 2014) indica que os principais sintomas apresentados por pacientes depressivos são humor deprimido na maior parte do dia, interesse ou prazer acentuadamente diminuídos por todas ou quase todas as atividades, perda ou ganho significativo de peso sem estar em dieta, diminuição ou aumento do apetite, insônia ou hipersonia, agitação ou retardo psicomotor, fadiga ou perda de energia, sentimento de inutilidade ou culpa excessiva ou inadequada, capacidade diminuída de pensar ou concentrar- se ou indecisão e pensamentos de morte recorrentes, ideação suicida sem um plano específico, tentativa de suicídio ou plano específico para cometê-lo. (CREMASCO; BAPTISTA, 2017).

Teruel, Martínez e León (2014) apresentaram também que a desesperança, um modo negativo de crenças e expectativas sobre sua própria vida e futuro, é um sinal altamente perceptível as pessoas do seu convívio. A dificuldade de lidar com problemas e impulsividade aumentam o rico de atitudes contra a própria vida.

As práticas do suicídio podem ser explicadas por inúmeros fatores apontados na literatura que estão associados com uma concepção depressiva, comprovando ser esse um fenômeno multifatorial ou multidimensional. Fatores mais subjetivos como impulsividade, agressividade, percepção do corpo, desesperança, dificuldades de comunicação e falta de pertencimento social vêm sendo abordados por desencadearem o processo da idealização suicida que estão acometendo milhões de vidas ao redor do mundo. (SANTOS et al., 2017)

\section{$\underline{\text { Universitários como Grupo de Risco }}$}

O suicídio é a segunda maior causa de mortes entre os universitários, estimativas afirmam que cerca de um milhão de pessoas comentem suicídio a cada ano no mundo, 
equivalente a uma morte por suicídio a cada 40 segundos (TERUEL; MARTÍNEZ; LEÓN, 2014).

Nos estudos de Molina, Galvis e Arenas (2014) fatores como a separação do núcleo familiar, aumento das responsabilidades e reavaliação das atividades, que devem ser enfrentados em especial por universitários, podem causar tais instabilidades emocionais e físicas, tendo como consequência altos níveis de ansiedade. Com isso passou a ser identificado a suscetibilidade dos universitários a adquirirem depressão e a suicidar-se.

Um relatório realizado com estudantes universitários dos Estados Unidos da América (EUA) sobre o comportamento suicida, apontou que 3,7\% dos participantes idealizaram o suicídio nos últimos 12 meses e 1,5\% nas últimas duas semanas. A pesquisa ainda demonstrou o quanto às tentativas de suicídio que $0,8 \%$ dos estudantes a realizaram nos últimos 12 meses, 0,3\% nas últimas duas semanas e 0,2\% nos últimos dias. (SANTOS et al., 2017). Outros estudos também demonstram dados alarmantes, inclusive no Brasil:

Um estudo desenvolvido na Colômbia com 258 universitários, evidenciou que $31 \%$ apresentou ideação suicida. Já uma pesquisa realizada no nordeste do Brasil obteve, dentre os 637 estudantes universitários, uma prevalência de 7,5\% para a tentativa de suicídio e $52,5 \%$ para a ideação suicida. (SANTOS et al., 2017, p.2).

Intensificando os fatores de risco, ainda há desordens de abuso de substâncias, e perturbações de conduta alimentaria. Sendo assim, existem evidências de maior prevalência de quadros depressivos entre estudantes universitários em comparação com a população geral, como também há um aumento igual nas taxas de tentativas de suicídios e de mortes consumadas em jovens. (BAADER et al. 2014)

Outro fator mencionado por Pereira e Cardoso (2015) é que, universitários que fazem o curso de sua escolha e empatia apresentam níveis apresentam níveis mais baixos de depressão, distinguindo daqueles que cursam uma graduação por pressão familiar ou devido á capacidade de encontrar emprego no futuro. Um outro ponto importante determinado por Santos et al. (2017) foi que o exercício da prática religiosa como por exemplo, orar, meditar e outras manifestações de crença, ajudam a estabelecer o equilíbrio de sentimentos e emoções.

Portanto, essa situação representa um desafio às universidades, dificultando a realização de seu dever, exemplificado pela influência significativa dessas patologias no 
desempenho estudantil dos universitários mencionado por Baader (2014), cabendo a instituição desenvolver diagnósticos e medidas de intervenção.

Dado esse quadro Cremasco e Baptista (2017) afirmam que é fundamental a realização de estudos que tenham como objetivo mensurar os aspectos positivos da vida. Por isso detectar os fatores associados ao suicídio em universitários se torna fundamental, podendo ser associado posteriormente a medidas profiláticas como a identificação e avaliação dos fatores cognitivos que ajudam as pessoas a serem mais resistentes as distintas dificuldades da existência.

\section{$\underline{\text { Fatores de Riscos Associados ao Suicídio em Universitários }}$}

Foram múltiplos os fatores encontrados na revisão literária sobre a idealização suicida, sendo os principais fatores associados ao suicídio: depressão 100\%; transtornos psíquicos 64\%; abuso de substâncias ilícitas 37\%; desesperança 28\%; abuso sexual 10\% (Gráfico 1). Tendo uma ligação direta com o público mais susceptível a esse problema, que são as mulheres e os autodeclarados LGBT, já que estes são os que mais adquirem psicopatologias, em especial a depressão e estão mais vulneráveis a abuso e assédio sexual.

\section{Gráfico 1: Principais Fatores Associados ao Suicídio em Universitários}




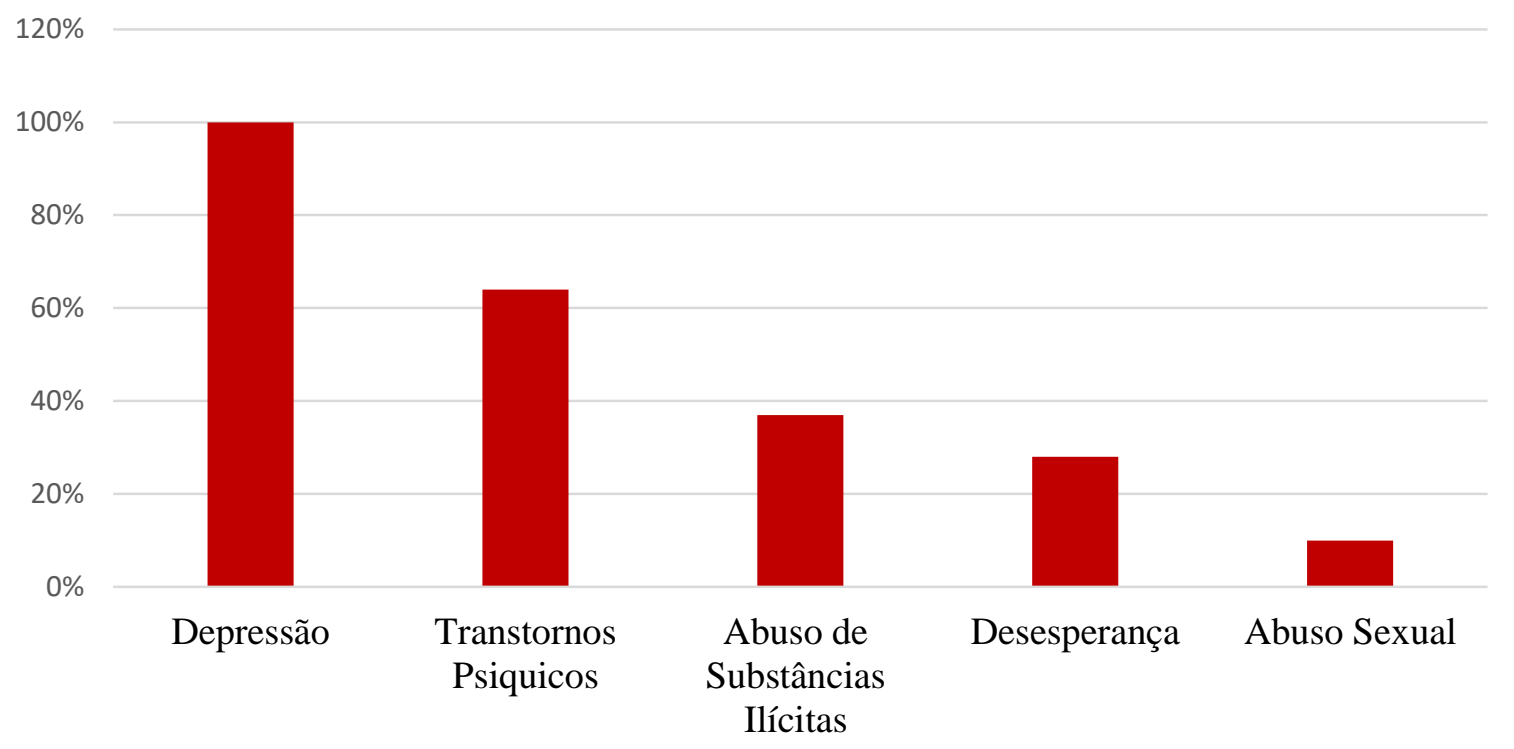

Segundo Teruel, Martínez e León (2014), com uma amostra de 40 estudantes do curso de Licenciatura em Psicopedagogia de uma Universidade do sul da Espanha, foi obtida uma amostra de 19 pessoas com ideação suicida, caracterizando 33,3\% ao total. Os fatores mencionados foram a depressão e a desesperança associado ao suicídio. Na pesquisa de Cremasco, Baptista (2017) que foi realizada entre 77 alunos de uma Universidade privada em uma cidade localizada no interior de Minas Gerais, obteve o mesmo padrão associado com a depressão.

Molina et al. (2014) mostra a partir de 39 estudantes do ensino superior com baixo rendimento da universidade de San Buenaventura da cidade de Medellín, que 10,3\% apresentaram critérios de depressão maior e risco de suicídio. Os de menor prevalência, representados por $2,6 \%$ cada um, foram distimia, transtorno bipolar, transtorno obsessivo compulsivo, transtorno de ansiedade generalizada, dependência de álcool, TDAH e transtorno de estres pós-traumático mais consumo de álcool e substancias psicoativas. Isso possui uma influência direta no aumento no risco de suicídios.

Os autores Cardoso e Pereira (2015) realizaram estudos em uma população universitária com 366 alunos participantes, em uma Universidade particular localizada norte de Portugal. O principal fator levantado foi a ideação suicida, estabelecendo que a cada um em dez estudantes experimentaram ideação suicida significativa. Baader et al. (2014), obteve um resultado semelhante, com um destaque a 18,3\% das mulheres que apresentaram 
depressão maior, um número elevado se comparado ao de homens $(9,7 \%)$. O estudo também revela uma alta prevalência de outras psicopatologias, sendo um grupo de risco para problemas de maior complexidade. A depressão é possivelmente um elemento central no risco do suicídio.

Em outro estudo, desenvolvido por Faria, Gandolfi e Moura (2014), foram analisados 210 jovens, de uma instituição de ensino superior em Brasília, observando que 9,4\% das mulheres apresentaram tentativas de suicídio, um alto contraste com a porcentagem masculina (1,2\%). Santos et al. (2017) realizou seu trabalho na Universidade Federal de Mato Grosso UFMT, Brasil, com 637 estudantes. 9,9\% dos estudantes responderam que nos últimos 30 dias pensaram em se matar.

Hawley et al (2016) obteve em uma universidade pública do meio oeste dos Estados Unidos um total de 1969 amostras analíticas. Foi consistida de três secções, 1- física, mental e estado geral de saúde; 2- conhecimento e experiências de suicídio; e 3- Dados pessoais do entrevistado, e os que apresentaram maior aspecto depressivo tinham maior risco de suicídio. Para tentar entender o suicídio, Franco e colaboradores (2017) realizaram um exercício teórico aplicado em 66 participantes. Foram relatados que dos 45 casos de suicídios, 68,9\% eram do sexo masculino e $31,1 \%$ do sexo feminino

O estudo de Cuesta e colaboradores (2015) foi efetivado com 100 estudantes entre 18 e 24 anos de uma Universidade particular da cidade de Medellín. Abordando um questionamento que incluía informação sociodemográfica, áreas de relações, condutas relacionadas com o aspecto suicida e que poderiam colocar a vida em risco. Foi constatado que cerca de $16 \%$ dos estudantes relataram ter tido ideação suicida, sendo a maior taxa em estudantes do sexo feminino com menos de 21 anos.

Sepúlveda, Pérez e Valencia (2016) observaram em um total de 1265 alunos de um programa de Psicologia de uma Universidade pública colombiana, através de um questionamento com fatores sociodemográfica, problemas de saúde, se tinham praticado automutilação e a quantidade te vezes que praticava; que 66,7\% dos homens declararam tentativas de suicídio e 48,5\% das mulheres. Foi identificado que 4,1\% das mulheres apresentaram um alto risco suicida. No total 3,6 \% dos entrevistados apresentaram um alto risco suicida. Aqueles pacientes que tendem a sofrer mais com a depressão e a ansiedade são predispostos a tentativas de suicídio. 


\section{PROPEX}

Sendo a depressão o principal fator para o suicídio, os fundamentais desencadeadores da depressão em universitários encontrados na análise de literatura foram: Pressão devido ao ambiente acadêmico (100\%), questões socioeconômicas 46\%, sexualidade 37\%, religião (falta de religião ou aspectos espirituais) $19 \%$ e problemas de saúde $10 \%$, como ilustrado no Gráfico 2.

\section{Gráfico 2: Principais Fatores Desencadeadores da Depressão em Universitários}

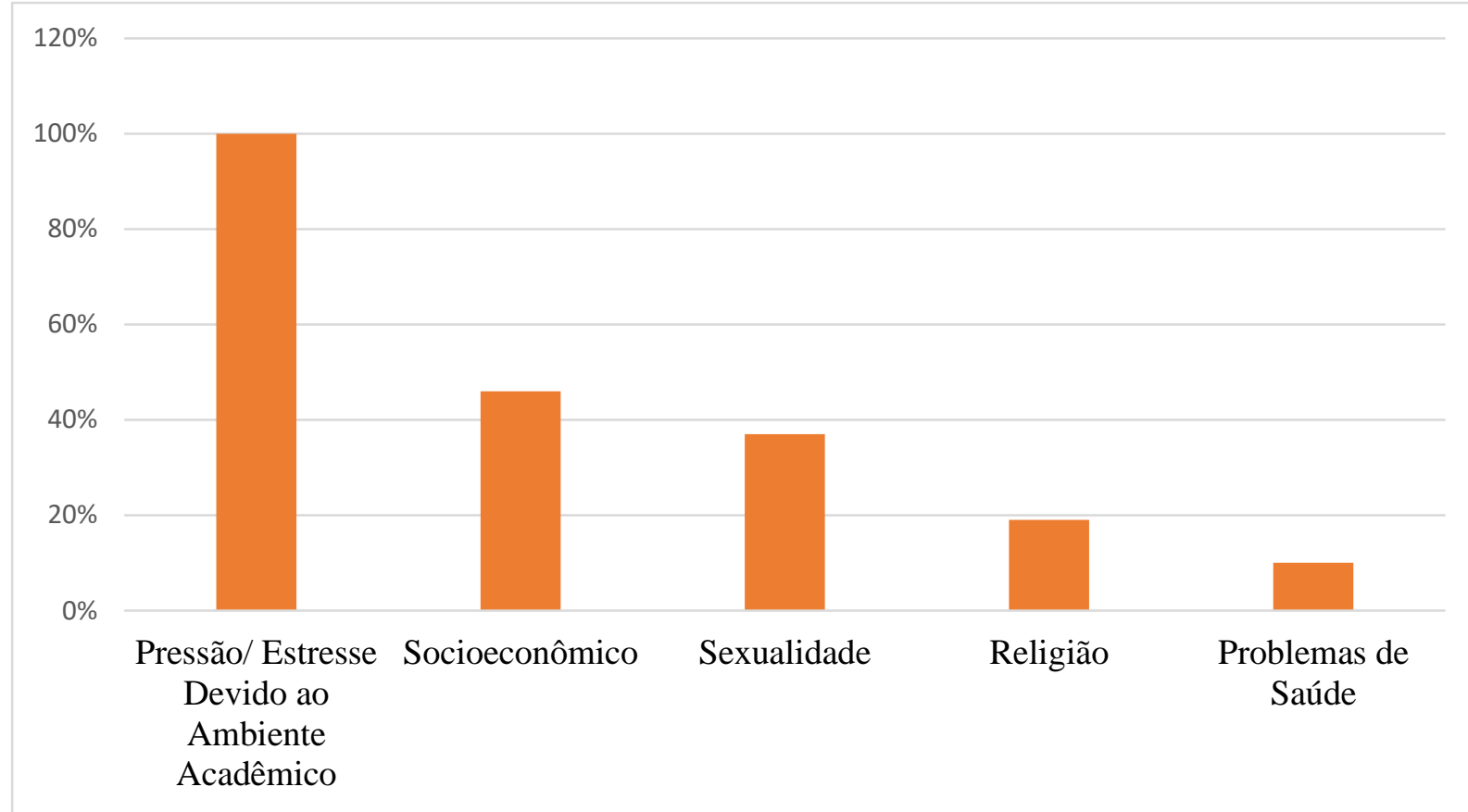

Na pesquisa de Teruel, Martínez e León (2014), a depressão é explicada pelo alto nível de estresse acadêmico é fundamental situação de risco para cometer uma tentativa. Outros fatores pertinentes são os socioeconómicos, epigenéticos, familiares, culturais e educativos. De acordo com as respostas analisadas por Cremasco e Baptista (2017) foram obtidos como principais categorias ao desencadeador do suicídio e depressão foram, dor/sofrimento, incapacidade de lidar com problemas, fuga.

Baader et al. (2014), apresenta uma associação da depressão com transtornos alimentares, consumo prejudicial de álcool e o risco de suicídio. Este risco de suicídio está relacionado a jovens com depressão maior e altos níveis de desesperança. No estudo, desenvolvido por Faria, Gandolfi e Moura (2014), relaciona questões como o consumo de 
drogas, álcool, ansiedade generalizada, abuso sexual foram mencionados como fatores relacionados a depressão e ao risco de suicídio.

O estudo de Santos et al. (2017) mostra as mulheres, assim como os homossexuais e os bissexuais, e os que não tinham uma prática religiosa, e uma associação do uso de álcool, com o desenvolvimento da depressão, e os indivíduos que possuíam níveis econômicos mais baixos apresentaram maior prevalência de ideação suicida. E os trabalhos de Hawley et al (2016), e Cuesta e colaboradores (2015), reforçam esses fatores relacionados a sexualidade relacionando a um menor entusiasmo com a sua saúde mental, física e estado geral de saúde.

\section{CONSIDERAÇÕES FINAIS}

Os tumultos da sociedade moderna provocam um aumento nos níveis de estresse e ansiedade dos indivíduos, que por muitas vezes levam ao surgimento da depressão principalmente nos jovens, em destaque os universitários. Por isso cada vez mais pesquisadores tem buscado identificar os fatores associados ao suicídio em discentes de instituições de ensino superior.

Esta revisão bibliográfica mostrou que o preconceito, estresse acadêmico, questões socioeconômicas, distância da família, desencadeiam um aumento nos níveis de ansiedade e melancolia, levando muitas vezes ao maior consumo de álcool e outras drogas, agravando ainda mais o quadro depressivo.

Então, observou-se que o aumento desses fatores de risco a saúde, que levam a depressão juntamente com os elementos estressores levam ao suicídio, caracterizando uma das principais causas de morte em universitários. Ainda foi possível concluir que, em média, há um público mais susceptível ao suicídio e a depressão no âmbito acadêmico, caracterizado por mulheres e autodeclarados LGBT.

A ausência de religiosidade, a saúde no geral e abuso sexual foram outros pontos que identificamos como desencadeadores de problemas psíquicos e idealização suicida no ambiente universitário, sendo algo que muitas vezes passa desapercebido pelos próprios estudantes e instituições de ensino superior. Todos os aspectos supracitados, quando não levam a efetividade do suicídio, acarretam dificuldades secundárias, como o baixo desempenho acadêmico, caracterizando um ciclo de frustações e problemas. 
Por isso, torna-se imprescindível as universidades e a sociedade no geral o conhecimento desses fatores associados ao suicídio de graduandos, pois assim será possível realizar a identificação desses itens e assim realizar medidas de combate e prevenção, melhorando o ambiente acadêmico, social e a saúde como um todo. Para tanto, as pesquisas nessa vertente ainda são escassas, em destaque nas áreas de investigação de tais fatores e de projetos no tratamento e profilaxia dos universitários.

\section{REFERÊNCIAS BIBLIOGRÁFICAS}

BAADER M, T. et al. Diagnóstico de la prevalencia de trastornos de la salud mental en estudiantes universitarios y los factores de riesgo emocionales asociados. Rev. chil. neuropsiquiatr., Santiago , v. 52, n. 3, p. 167-176, sept. 2014. Disponível em: <http://www.scielo.cl/scielo.php?script=sci_arttext\&pid=S0717-

92272014000300004\&lng=es\&nrm=iso>.

Acesso

em: 28 Jul 2017. http://dx.doi.org/10.4067/S0717-92272014000300004.

BLANDON CUESTA, O. M. et al. Ideación suicida y factores asociados en jóvenes universitarios de la ciudad de Medellín. AMC, Camagüey, v. 19, n. 5, p. 469-478, oct. 2015. Disponível em: <http://scielo.sld.cu/scielo.php?script=sci_arttext\&pid=S102502552015000500006\&lng=es\&nrm=iso>. Acesso em: 28 Jul 2017

CREMASCO, G. S.; BAPTISTA, M.N. Depressão, motivos para viver e o significado do suicídio em graduandos do curso de psicologia. Estudos Interdisciplinares em Psicologia, $\begin{array}{llllllll}\text { Londrina, } & \text { v. } & \text { 8, } & \text { n. } & 1, & \text { p. 22-37, jun. } 2017 & \text { Disponível }\end{array}$ em: $\langle$ http://www.uel.br/revistas/uel/index.php/eip/article/view/24293/20138>. Acesso em: 28 Jul 2017.

FARIA, Y. O.; GANDOLFI, L.; MOURA, L. B. A. Prevalência de comportamentos de risco em adulto jovem e universitário. Acta paul. enferm., São Paulo , v. 27, n. 6, p. 591595, Dec. 2014. Disponível em: $<$ http://www.scielo.br/scielo.php?script=sci_arttext\&pid=S0103- 
$21002014000600591 \& \operatorname{lng}=\mathrm{en} \& \mathrm{nrm}=\mathrm{iso}>$.

Acesso

em: 28 Jul 2017.http://dx.doi.org/10.1590/1982-0194201400096.

FRANCO, S. A. et al. Suicidio en estudiantes universitarios en Bogotá, Colombia, 20042014. Ciênc. saúde coletiva, Rio de Janeiro ， v. 22, n. 1, p. 269-278，Jan. 2017. Disponível em: $<$ http://www.scielo.br/scielo.php?script=sci_arttext\&pid=S1413-

$81232017000100269 \& \operatorname{lng}=\mathrm{en} \& \mathrm{nrm}=$ iso $>$.

Acesso

em: 28 Jul 2017. http://dx.doi.org/10.1590/1413-81232017221.22452015.

HAWLEY, L.D. et. al. Baseline assessment of campus-wide general health status and mental health: Opportunity for tailored suicide prevention and mental health awareness programming. Journal of American College Health, Disponível em: < http://dx.doi.org/10.1080/07448481.2015.1085059> Acesso em: 28 Jul. 2017 DOI: $10.1080 / 07448481.2015 .1085059$

PEREIRA, A.; CARDOSO, F. Suicidal Ideation in University Students: Prevalence and Association With School and Gender. Paidéia (Ribeirão Preto), Ribeirão Preto, v. 25, n. 62, p. 299-306,

Dec. 2015.

Disponível

em: $<$ http://www.scielo.br/scielo.php?script=sci_arttext\&pid=S0103863X2015000300299\&lng=en\&nrm=iso>. Acesso em: 28 Jul 2017. http://dx.doi.org/10.1590/1982-43272562201503.

SANTOS, H. G. B. et al. Factors associated with suicidal ideation among university students. Rev. Latino-Am. Enfermagem, Ribeirão Preto, v. 25, e2878, 2017. Disponível em: $<\mathrm{http}: / /$ www.scielo.br/scielo.php?script=sci_arttext\&pid=S010411692017000100332\&lng=en\&nrm=iso>. Acesso em: $28 \mathrm{Jul}$ 2017. Epub May 15, 2017. http://dx.doi.org/10.1590/1518-8345.1592.2878.

SEPULVEDA, P. C. G.; PEREZ, O. A. M.; VALENCIA, J. C. O. Riesgo suicida y factores asociados en estudiantes de Psicología en una Universidad pública de Colombia. Revista Habanera de Ciencias Médicas, v.15, n.1, feb. 2016.

Disponível 
em:<http://scielo.sld.cu/scielo.php?script=sci_arttext\&pid=S1729-

519X2016000100015\&lng=es\&nrm=iso>. Acesso em: 28 Jul 2017.

TERUEL, D. S.; MARTÍNEZ, J. A. M.; LEÓN, A. G. Variables psicológicas asociadas a la ideación suicida en estudiantes. International Journal of Psychology and Psychological Therapy, 2014, 14, 2, 277-290. Disponível em: http://www.ijpsy.com/volumen14/num2/388.html Acesso em: 28 Jul 2017.

VALENCIA, A.M.M.; GALVIS, A.M.P.; ARENAS, D.A.M. Trastornos neuropsiquiátricos en estudiantes universitarios con bajo rendimiento académico de una universidad privada de Medellín. CES Psicología, 7, 1, 69-78. Disponível em: <http://revistas.ces.edu.co/index.php/psicologia/article/view/2766>. Acesso em: 28 Jul 2017. 